\title{
Improving parent information in neonatal care: A ward-based co-design activity with parents to improve satisfaction
}

\section{Imperial College London}

\author{
$\underline{\text { S. Sakonidou }}{ }^{1}$, S. Kotzamanis ${ }^{2}$, I. Andrzejewska ${ }^{1}$, W. Carnegie ${ }^{3}$, M. Patel ${ }^{4}$, A.J. Poots ${ }^{5}$, M.A Nakubulwa, ${ }^{3}$ T. Woodcock ${ }^{3}$, N. Modi ${ }^{1}$, D. Bell ${ }^{3}$, C. Gale ${ }^{1}$ \\ ${ }^{1}$ Imperial College London, Department of Medicine, London, United Kingdom. \\ ${ }^{2}$ Imperial College London, Parent representative, London, United Kingdom. \\ ${ }^{3}$ National Institute for Health Research Collaboration for Leadership in Applied Health Research and Care, Northwest London, London, United Kingdom. \\ ${ }^{4}$ BLISS for babies born premature or sick, The National Charity for the Newborn UK, London, United Kingdom. \\ ${ }^{5}$ Independent researcher, Oxford, Oxford, United Kingdom.
}

\section{Background and aims}

1 in 8 babies born in the UK are admitted for neonatal care; a highly stressful time for parents. When their baby is admitted to a neonatal unit parents are commonly provided with written information, traditionally designed by clinical staff. This study describes parents and clinician co-design of information provision in neonatal care. We hypothesised this would improve parent satisfaction with information received.

\section{Methods}

We recruited parents with experience of UK neonatal care. The study was co-led by our parent representative and had 4 stages:

Parents of ex-neonatal babies discussed their experience of information sharing in neonatal care I. Exploration and their information needs.

We used parent feedback from stage 1 to re-design the existing clinician-designed

2. Co-design information (booklet A) provided to parents on admission to a tertiary neonatal unit and created booklet $B$.

3. Evaluation We used quality improvement methodology to refine the information based on parent feedback on the neonatal unit (booklet $\mathbf{C}$ ).

4. Reevaluation

We assessed parent satisfaction with information booklets $\mathrm{A}, \mathrm{B}$, and $\mathrm{C}$ using a tailor made questionnaire, covering 3 themes: Content, Format and Overall Satisfaction (5 point Likert scale, 1 least satisfied -5 most satisfied).

Booklet A $\rightarrow$ B $\rightarrow$ C

Results

Stage 1

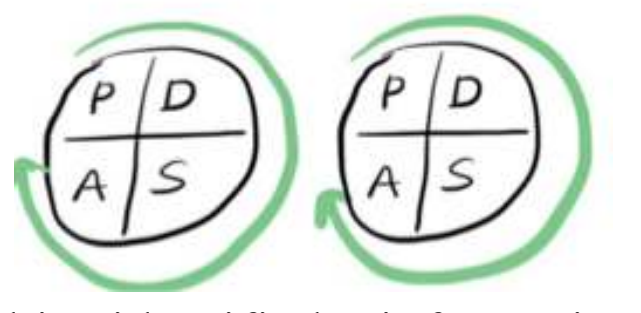

Parents of ex-neonatal babies identified 7 information themes as important:

(i) Clinical updates

(ii) Neonatal unit rules and policies

(iii) Equipment

(iv) Infant location on the neonatal unit

(v) Parent support

(vi) Parent involvement and empowerment

(vii) Infant transfer process to other units/hospitals

Stages 2, 3, 4: Demographics of parents on neonatal unit Stages 2-4 were conducted over 2 weeks with 26 parents on the neonatal unit. 14 parents evaluated booklet A, 19 parents booklet B and 11 parents booklet C. Parents evaluated booklets A, B or C according to when they were approached and how long they remained on the unit for. $4 / 26$ parents evaluated all booklets A, B and C. The majority of parent participants were mothers $(70.5 \% \mathrm{vs}$ $29.5 \%$ fathers). Most parents had been on the unit for 1 week- 1 month $(40.9 \%)$ and their babies were born at $24-30$ weeks $(34.1 \%)$.
Theme 1: Content of booklet

Does the booklet contain enough

information about how the neonatal unit works?

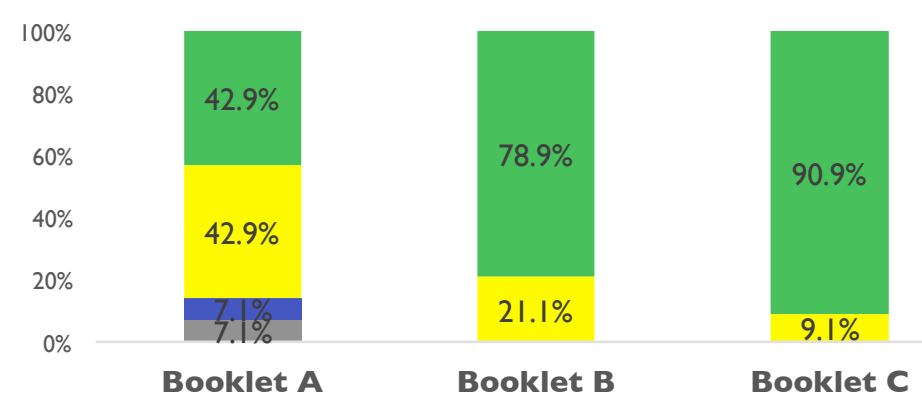

Does the booklet explain the purpose of the machines, monitors and alarms/"beeping" in the neonatal unit?
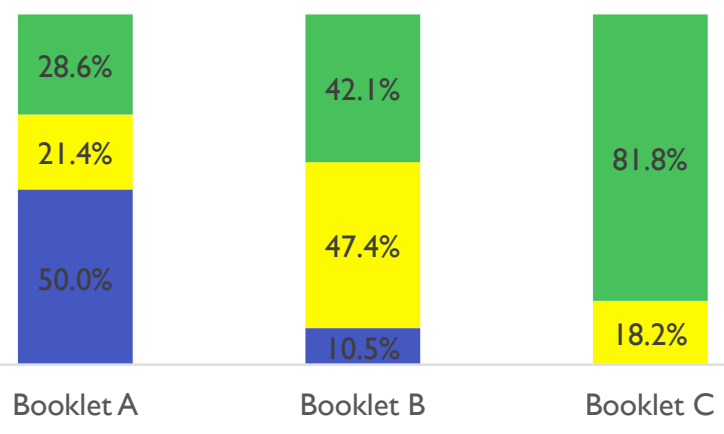

Does booklet contain enough information about being involved in your baby's care?
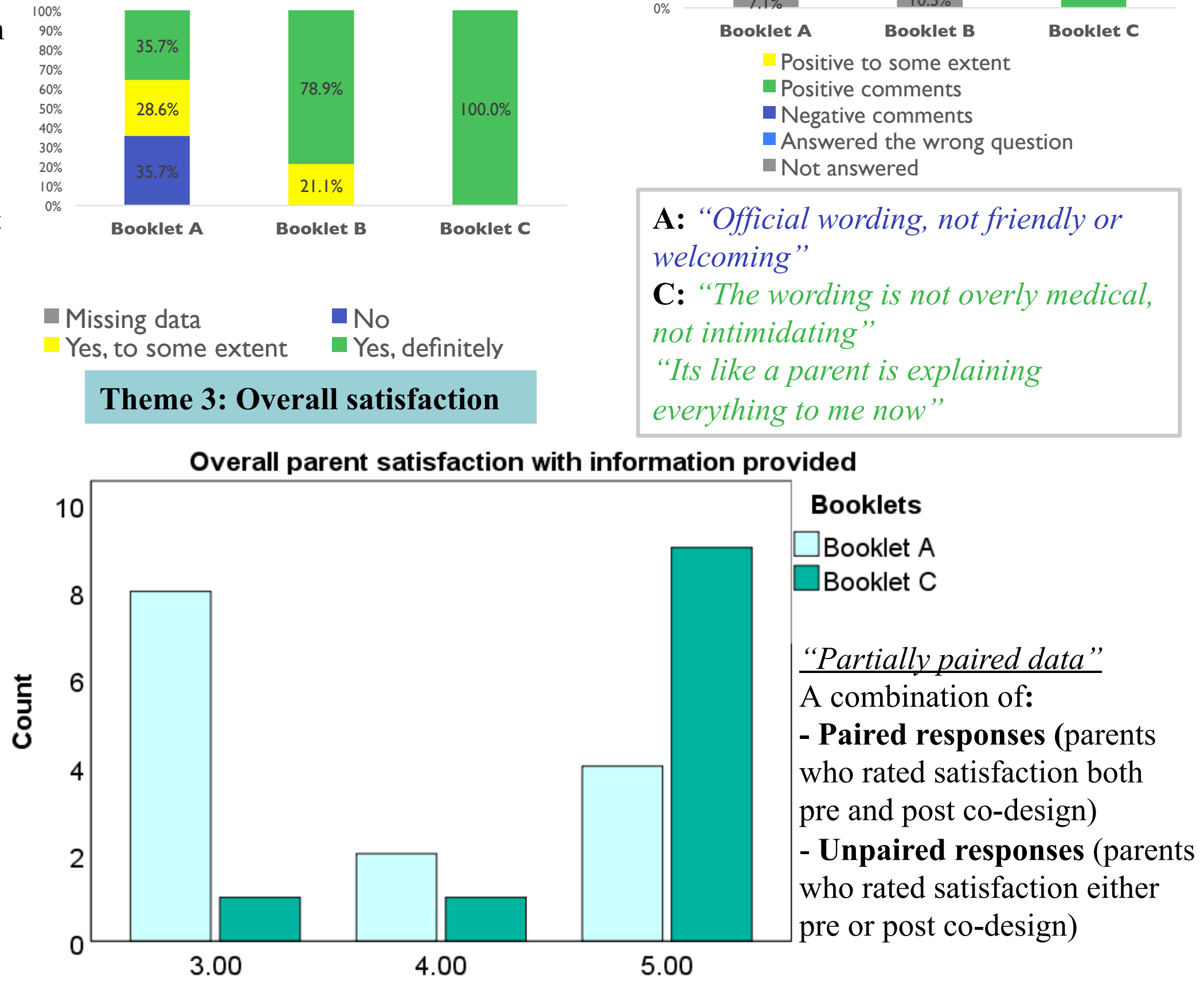

Q6:On a scale of 1 to 5 how satisfied were you with the information provided to you in the booklet?

- We analysed this partially paired data using the optimal pooled t-test $\left(\mathrm{T}^{0}{ }_{\text {pool }}\right)$ as described by Guo \& Yuan (2017).

- On average, parents were significantly $(\mathrm{t} 12.0=-3.67, \mathrm{p}=0.00310)$ more satisfied with information provided in the parent-clinician co-designed Booklet $\mathrm{C}(\mathrm{M}=4.72)$ than they were with the clinician-designed Booklet A $(\mathrm{M}=3.71)$. Adjusting for the unpaired data, the estimate of the mean difference score was -1.08 on the Likert scale.
Theme 2: Format of booklet

in did you feel about the order of information in the booklet?

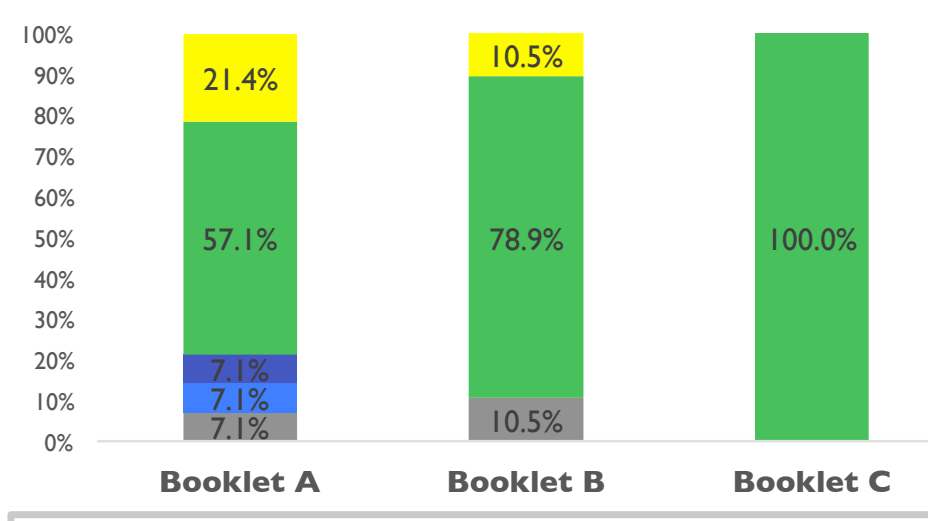

A: "The order of the information doesn't feel right"

C: "The order of the booklet works very vell and gives a great understanding of

How did you feel about the way information was worded in the booklet?

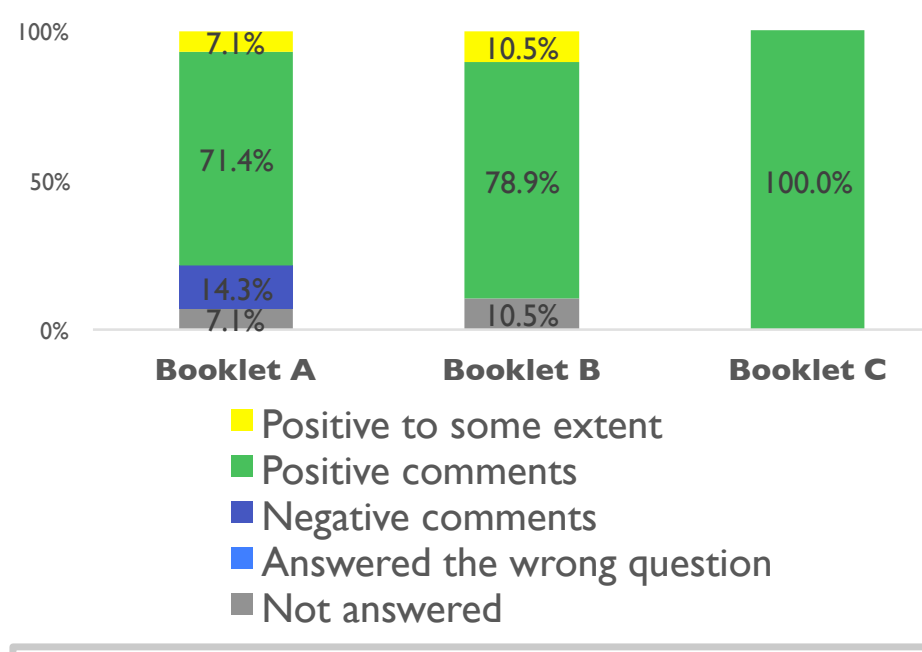

A: "Official wording, not friendly or welcoming'

C: "The wording is not overly medical, not intimidating"

"Its like a parent is explaining

everything to me now"

\section{Conclusions}

A co-designed neonatal care booklet improved parent satisfaction with communication of information in neonatal care. Parent co-design methodology provides potential for improving communication in neonatal care. 